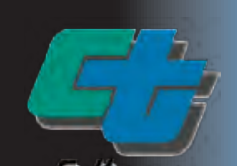

\title{
USGS ShakeCast
}

\section{Automating, Simplifying, and Improving the Use of ShakeMap for Post-Earthquake Decisionmaking and Response}

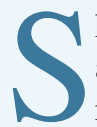

hakeCast is a freely available, post-earthquake situational awareness application that automatically retrieves earthquake shaking data from ShakeMap, compares intensity measures against users' facilities, and generates potential damage assessment notifications, facility damage maps, and other Web-based products for emergency managers and responders.

\section{What is ShakeCast?}

ShakeCast, short for ShakeMap Broadcast, is a fully automated system for delivering specific ShakeMap products to critical users and for triggering established post-earthquake response protocols. ShakeMap is a well-established tool used to portray the extent of potentially damaging shaking following an earthquake. ShakeMap is automatically generated for small and large earthquakes in areas where it is available and can be found on the Internet at http://earthquake.usgs.gov/shakemap/. It was developed and is used primarily for emergency response, loss estimation, and public information. However, for an informed response to a serious earthquake, critical users must go beyond just looking at ShakeMap, and understand the likely extent and severity of impact on the facilities for which they are responsible. To this end the U.S. Geological Survey (USGS) has developed ShakeCast.

ShakeCast allows utilities, transportation agencies, businesses, and other large organizations to control and optimize the earthquake information they receive. With ShakeCast, they can automatically determine the shaking value at their facilities, set thresholds for notification of damage states for each facility, and then automatically notify (by pager, cell phone, or email) specified operators and inspectors within their organizations who are responsible for those particular facilities so they can set priorities for response.

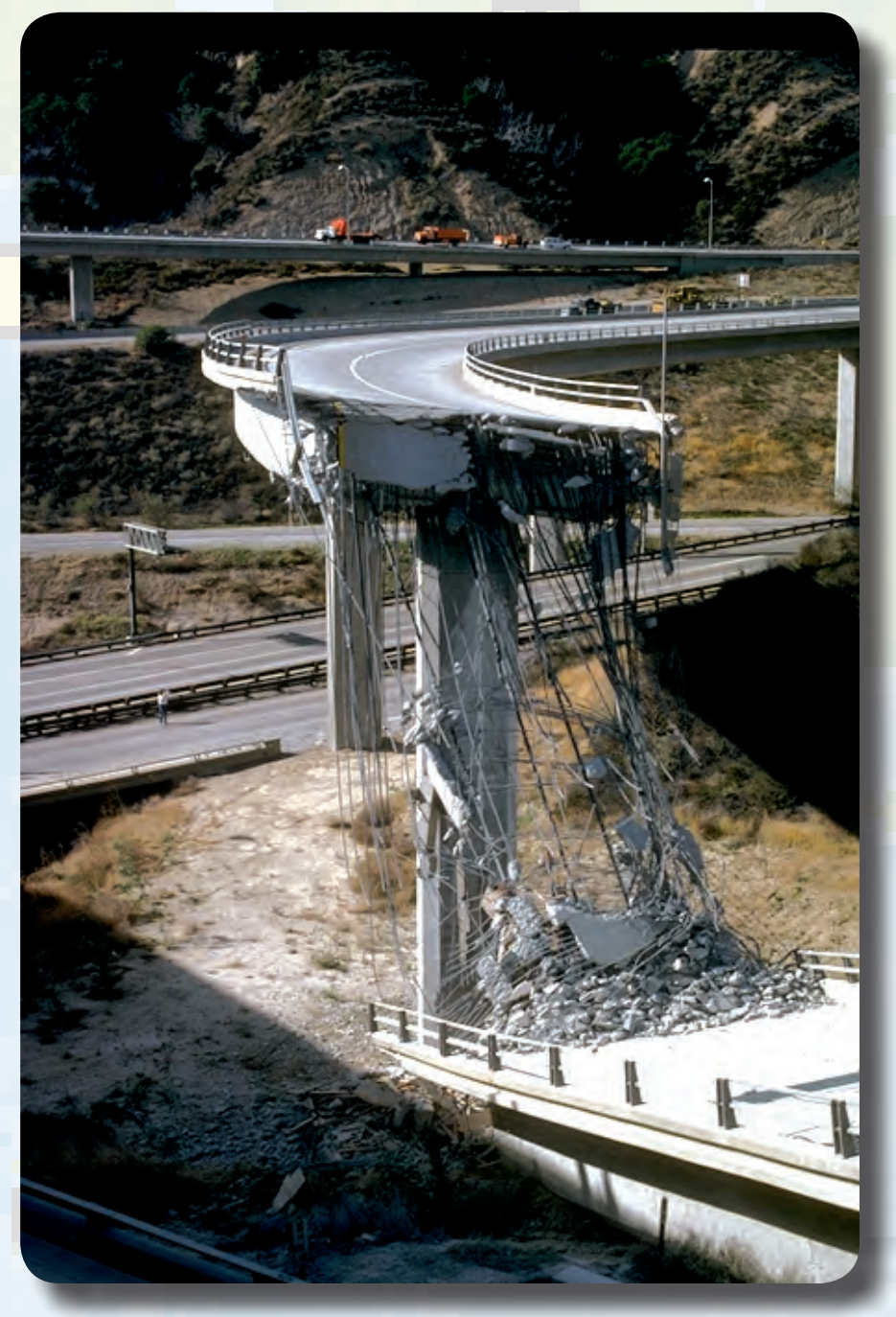

Collapse of the Interstate-5/State Highway-14 interchange showing damage north of Los Angeles caused by the 1994 magnitude 6.7 Northridge, California, earthquake. Thousands of State and County bridges were shaken at varying intensity levels during this earthquake; many required inspections.

\section{Example Uses and Users: The California Department of Transportation (Caltrans)}

Caltrans has deployed the prototype ShakeCast system (Version 1.0). Following a major earthquake, Caltrans faces an array of decisionmaking challenges. Perhaps no other agency has a comparable earthquake exposure in the State of California. Caltrans has more than 11,000 bridges and overpasses under its responsibility in California; having an instantaneous snapshot of the likely damage to each will allow Caltrans to set priorities for traffic rerouting, closures, and inspections following a damaging earthquake. One of several critical tasks facing Caltrans after an earthquake is to rapidly assess the condition of all bridges and roadway corridors in the State highway system. Timely response is important to ensure public safety, aid routing of emergency vehicle traffic, and (re-) establish critical lifeline routes. 
In addition to real-time notification, ShakeCast also can generate and deliver scenario earthquakes for facility response plans (figs. 1 and 2). This application includes routine testing of the system, earthquake scenario exercises, and evaluating performance and response under potential earthquake conditions. ShakeMap is now used routinely to generate earthquake scenarios for many regions; ShakeCast will further allow planning exercises to be performed using the same notification tools that will be available and in place for responding to a real earthquake.

\section{ShakeCast Technology}

Individuals, companies, utilities, and agencies could develop their own strategies and tools for using ShakeMap given their

\section{Scenario ShakeMap: Southern San Andreas Fault}

Scenario Date: Thu Nov 8, 2008 10:00:00 AM PDT M 7.8 N33.92 W116.47 Depth: 10.0km

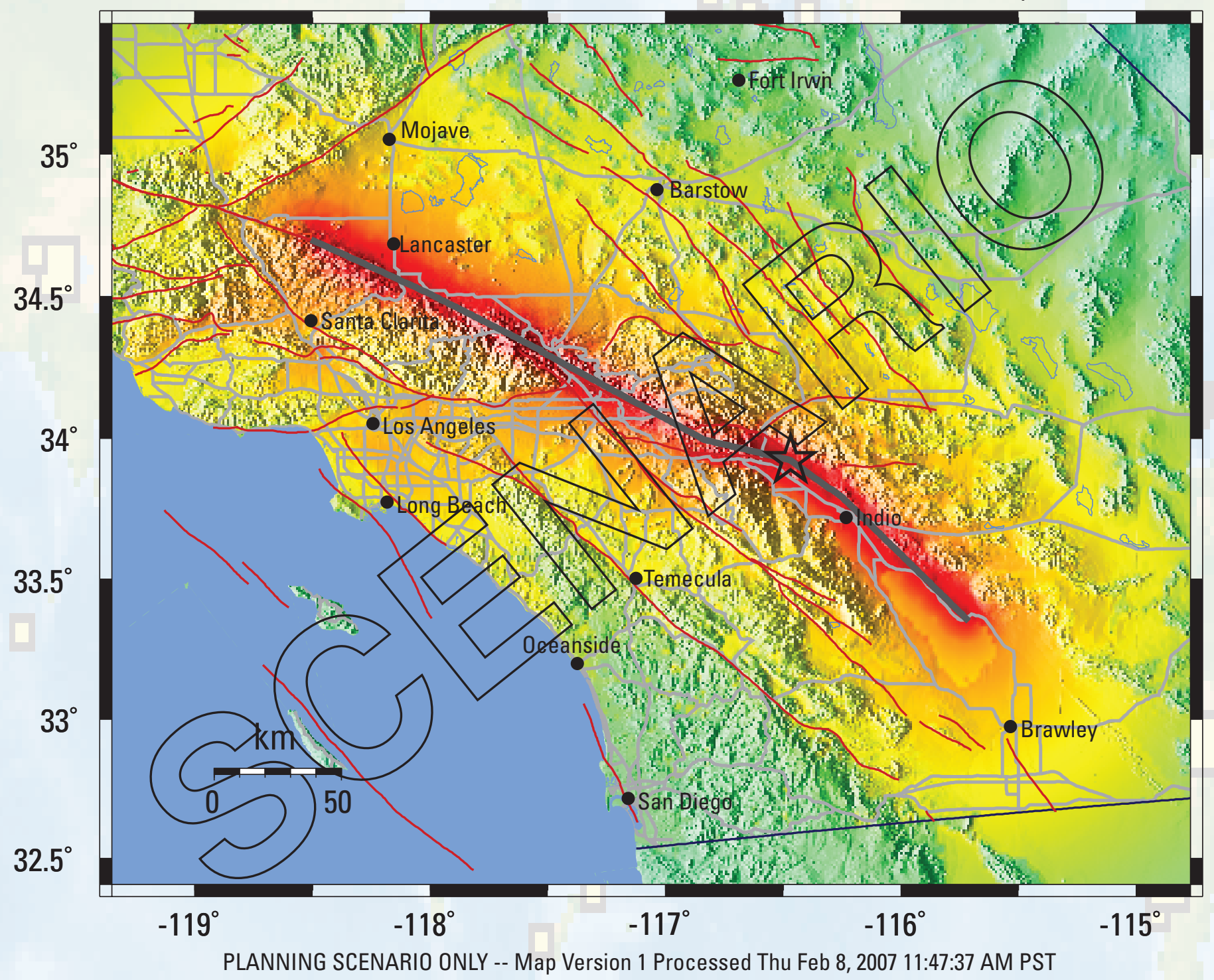

\begin{tabular}{|c|c|c|c|c|c|c|c|c|c|}
\hline $\begin{array}{c}\text { PERCEIVED } \\
\text { SHAKING }\end{array}$ & Not felt & Weak & Light & Moderate & Strong & Very strong & Severe & Violent & Extreme \\
\hline $\begin{array}{c}\text { POTENTIAL } \\
\text { DAMAGE }\end{array}$ & none & none & none & Very light & Light & Moderate & Moderate/Heavy & Heavy & Very Heavy \\
\hline PEAK ACC.(\%g) & $<.17$ & $\mathbf{. 1 7 - 1 . 4}$ & $\mathbf{1 . 4 - 3 . 9}$ & $\mathbf{3 . 9 - 9 . 2}$ & $\mathbf{9 . 2 - 1 8}$ & $18-34$ & $34-65$ & $65-124$ & $>124$ \\
\hline PEAK VEL.(cm/s) & $<0.1$ & $0.1-1.1$ & $1.1-3.4$ & $3.4-8.1$ & $\mathbf{8 . 1 - 1 6}$ & $\mathbf{1 6 - 3 1}$ & $\mathbf{3 1 - 6 0}$ & $\mathbf{6 0 - 1 1 6}$ & $>\mathbf{1 1 6}$ \\
\hline $\begin{array}{c}\text { INSTRUMENTAL } \\
\text { INTENSITY }\end{array}$ & $\mathbf{I}$ & $\mathbf{I I - I I I ~}$ & $\mathbf{I V}$ & $\mathbf{V}$ & $\mathbf{V I}$ & VII & VIII & IX & $\mathbf{X}+$ \\
\hline
\end{tabular}

Figure 1. ShakeMap intensity map for a magnitude 7.8 Scenario Earthquake on the southern San Andreas fault. Red lines delineate faults and light grey lines show highways. 


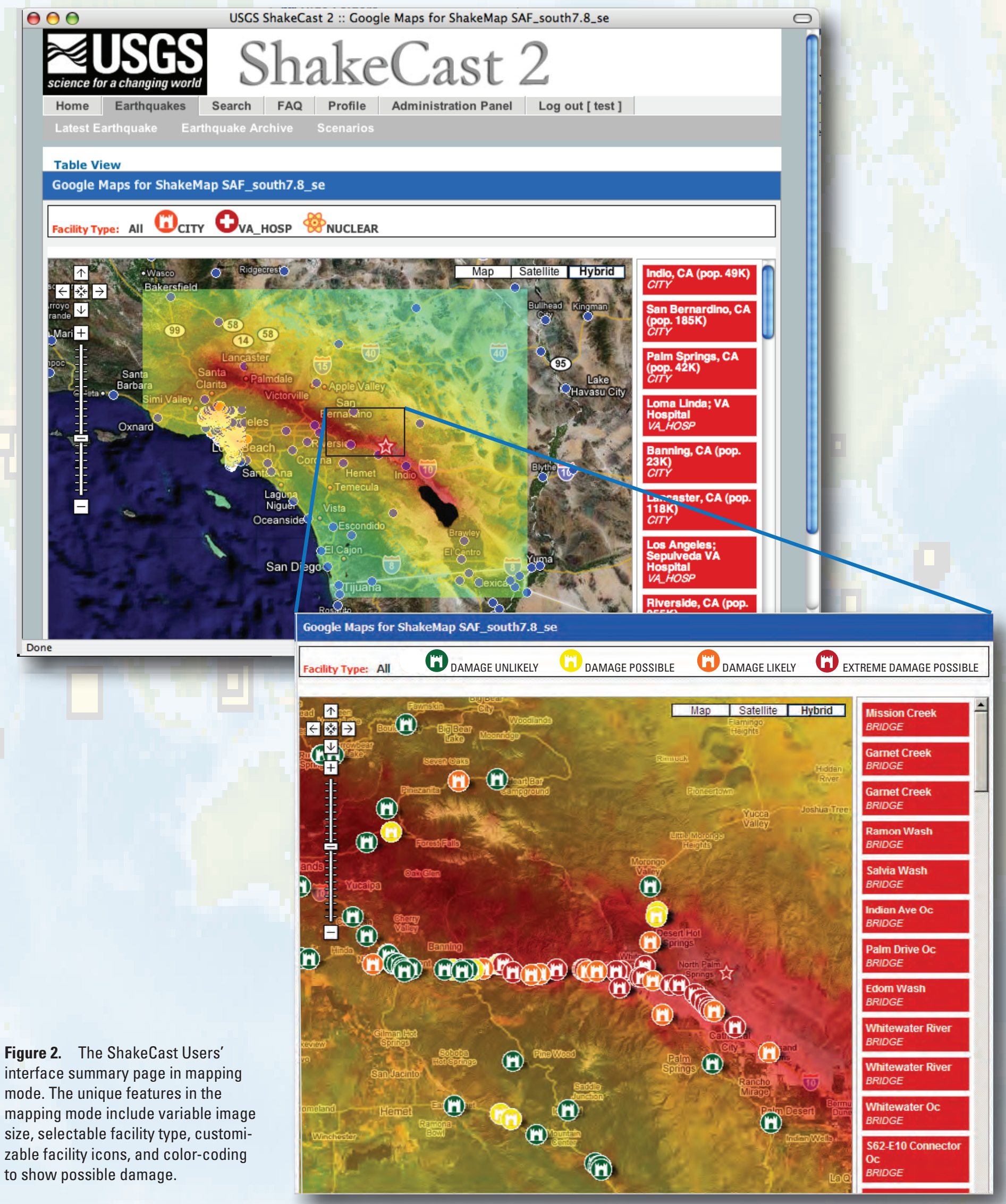




\section{Example Uses and Users: Los Angeles Unified School District (LAUSD)}

LAUSD is using Shakecast to help improve earthquake monitoring and emergency response in southern California, where it is responsible for over 700,000 K-12 students, 100,000 employees, 13,500 buildings, and 1,100 schools and offices. Students and schools are spread across 704 square miles. Under an agreement with the City of Los Angeles, LAUSD buildings are integral to the emergency operations of the city, because school facilities will serve as emergency shelters to be managed by the Red Cross. Hence, knowing which structures are most likely damaged is critical for response and recovery, for example, in designating emergency shelters.

unique facilities and communication paths. However, such efforts are costly and complex. Rather, the USGS is facilitating this process with ShakeCast, building a more general-use tool for the most critical user needs. The ShakeCast software is customizable for facilities, fragilities, and notifications, and we anticipate additional adaptations will be made since the opensource code is provided. Such innovations then can be provided in the tool kit included with updates of the ShakeCast system.

Information Technology (IT) security is a primary concern for users requiring automatic electronic delivery of information. By taking advantage of standard Internet protocols, ShakeCast users avoid most typical corporate and Government concerns and firewall limitations. By using Really Simply Syndication (RSS) and interval polling, users initiate all communications with the USGS Web servers that host ShakeMap, and retrieve selected products as a request rather than a "push." This RSS approach allows users to update software automatically under conditions of their own choosing.

ShakeCast software is built upon open-source tools, providing standard, freely available software for all users, encouraging user improvements, and simplifying interfacing with existing users' response tools. ShakeCast uses the Apache Web server and PHP (Hypertext Preprocessor) for dynamic Web content, MySQL for facility and notification databases, and is wrapped in Practical Extraction and Report Language (PERL) scripting. Exchange files are in Extensible Markup Language (XML) for standardized interfacing with Web, geographic information system (GIS), spreadsheets, databases, and other applications.

\section{Where Can ShakeCast be Used?}

ShakeMap is now produced for all earthquakes around the globe of magnitude 5.5 or larger. Globally, these ShakeMaps are primarily predictive and thus lack the resolution and certainty of shaking estimates for maps made in regions of dense seismic instrumentation for which it was principally developed. Regions in the United States that have ShakeMap operating with reasonable (but variable) seismic station coverage include major parts of California, Washington, Oregon, Nevada, Utah, Hawaii, and Alaska. Other regions are improving station coverage. Hence, since ShakeMaps are produced for any region of the world, ShakeCast can be deployed for any exposure of facilities worldwide, again with more uncertainty in the results in regions not specifically listed above.

We use the term "facilities" loosely; at the USGS National Earthquake Information Center (NEIC) in Golden, Colorado, we assign cities as "facilities" and run ShakeCast to determine shaking levels at cities within the United States and around the globe any time a ShakeMap is produced. The list of cities, their populations, and the intensity estimated at each city becomes a Hypertext Markup Language (HTML) email notification that proves useful for NEIC analysts and for other response purposes. Ultimately, these city-based notifications will be integrated as an option in the USGS Earthquake Notification Service (ENS), but this option does not reduce the need for critical users to put their own inventories into an in-house ShakeCast system.

\section{ShakeCast Availability and Installation}

ShakeCast is available in two levels, full and "Lite." We describe in detail the full ShakeCast system that allows users to estimate impact to numerous facilities, each potentially with different vulnerabilities and notification recipients. We expect this system to be deployed by critical users in an earthquakehardened, operational environment. We have also made available ShakeCast Lite, a subset of the system that allows users to automatically receive ShakeMap products on their laptop or desktop computers, and launch predefined applications using those maps or data. For example, many users employ ShakeCast Lite to automatically open a Web browser showing the latest ShakeMap in their region, launch Google Earth ${ }^{\circledR}$ with the ShakeMap KML file, download ShakeMap grid files, and initiate loss-estimation applications, or deliver ShakeMap GIS files to their corporate GIS department for further analyses. ShakeCast Lite is simple to install and use.

An overview of ShakeCast from the users' perspective is provided in figure 3. Organizations using ShakeMap/ShakeCast first download and install the ShakeCast (Version 2) software package on a hardened in-house computer system. The software is installed with an interactive installation script. Facility, vulnerability, and notification data are input using import tools and simple, comma separated (CSV) users' files. ShakeCast comes preconfigured, but custom configuration is simplified by ShakeCast tools and the Web interface. The Web interface allows an administrator to access all functions of the local ShakeCast system, and end users are able to manage their own personal information and notification preferences.

Initial setup involves the following steps: (1) populating a database of facility locations and types; (2) assigning fragilities using specific ShakeMap parameters (for example, intensity, peak or spectral acceleration) and the corresponding likely "green," "yellow," and "red" damage states ("damage unlikely," "damage possible," and "damage likely" thresholds, for example); (3) specifying who receives notifications by listing 
addresses of facility managers and response personnel (email, cell phone); and (4) selecting under which circumstances the alerts are sent (for example, damage "possible" at specific facilities). In addition, the user can customize the content of the summary report that is delivered internally; for example, a list of facilities based on their likely damage state, and organizational specific links and images.

Example user and earthquake data, tutorials, and documentation are provided with the installation package.

\section{Ongoing ShakeCast Development}

Ongoing software development of ShakeCast continues, and much of it is motivated by users' experiences and recommendations.
ShakeCast, Release Version 2.1, is expected to include the following enhancements:

- Additional predefined facility structure types and vulnerability functions. Currently (2007) 36 structure types are available from the Federal Emergency Management Agency's Hazards US loss modeling software (HAZUS-MH).

- Additional vulnerability types, including pipeline, liquefaction, and landslide failure potential. In addition to maps, 2-D profiles will provide pipeline cross-sectional views.

- Ability to select a specific structure and seismic instrument data over interpolated shaking values from ShakeMap.

Build Your Inventory Database Prior to Earthquakes

- Define regions of interest

- Collect structure information (location and fragility) or select from predefined structure types (right)

- Identify notification recipients, notification thresholds, and message formats

Automatically Receive the Earthquake Notification

- Alert from the ShakeCast system soon after an earthquake is located and a ShakeMap is created

- Alert message contains earthquake information and the number of facilities likely affected and to what degree - Quick emailed summary table (right) indicates estimated damage to facilites sorted according to likely impact

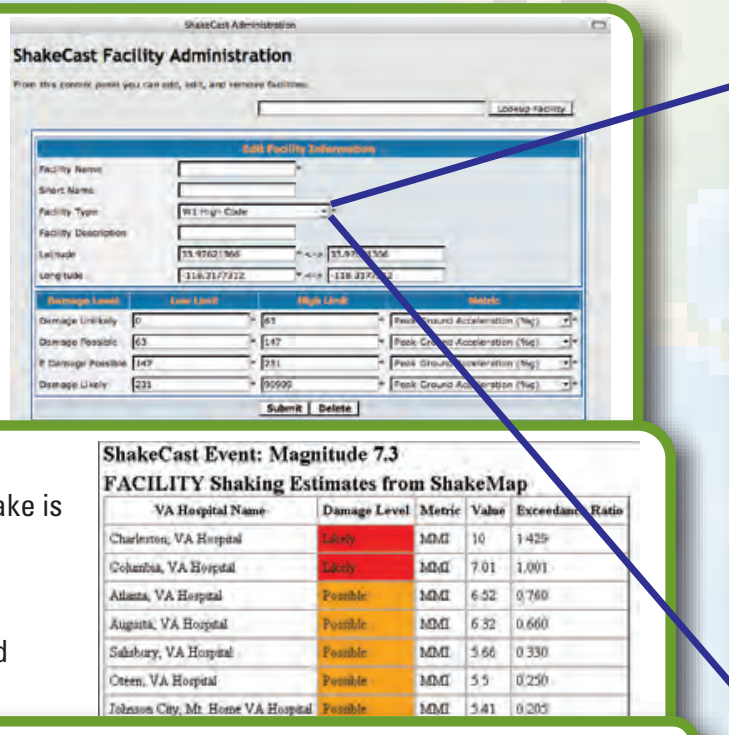

Multi-building campus
S5H Low Code
S5H Pre Code
S5L Low Code
S5L Pre Code
S5M Low Code
S5M Pre Code
URML Low Code
URML Pre Code
URMM Low Code
URMM Pre Code
W1 High Code
W1 Moderate Code
W1 Low Code
W1 Pre Code
W2 High Code
W2 Moderate Code
W2 Low Code
W2 Pre Code
Bridge
Multi-building campus

Check the Damage Assessment Estimate

- The ShakeCast Web interface (right) provides a quick summary of affected facilities, earthquake information, and Google Maps GIS tools

- Event table contains detailed information on groundshaking measures, facility information, and damage estimates

-The GIS interface integrates ShakeMap and users' facilities into categories for improved navigation and damage assessment; hot links can provide additional facility information

\section{Provide Updates for Post-Earthquake Response}

- ShakeCast system continues to receive ShakeMap updates and to provide updated prioritized list of facilities for inspection

- ShakeCast system automatically downloads selected ShakeMap products for organization-wide damage analysis

- ShakeCast system is capable of processing scenario earthquakes for the purpose of emergency planning and exercises
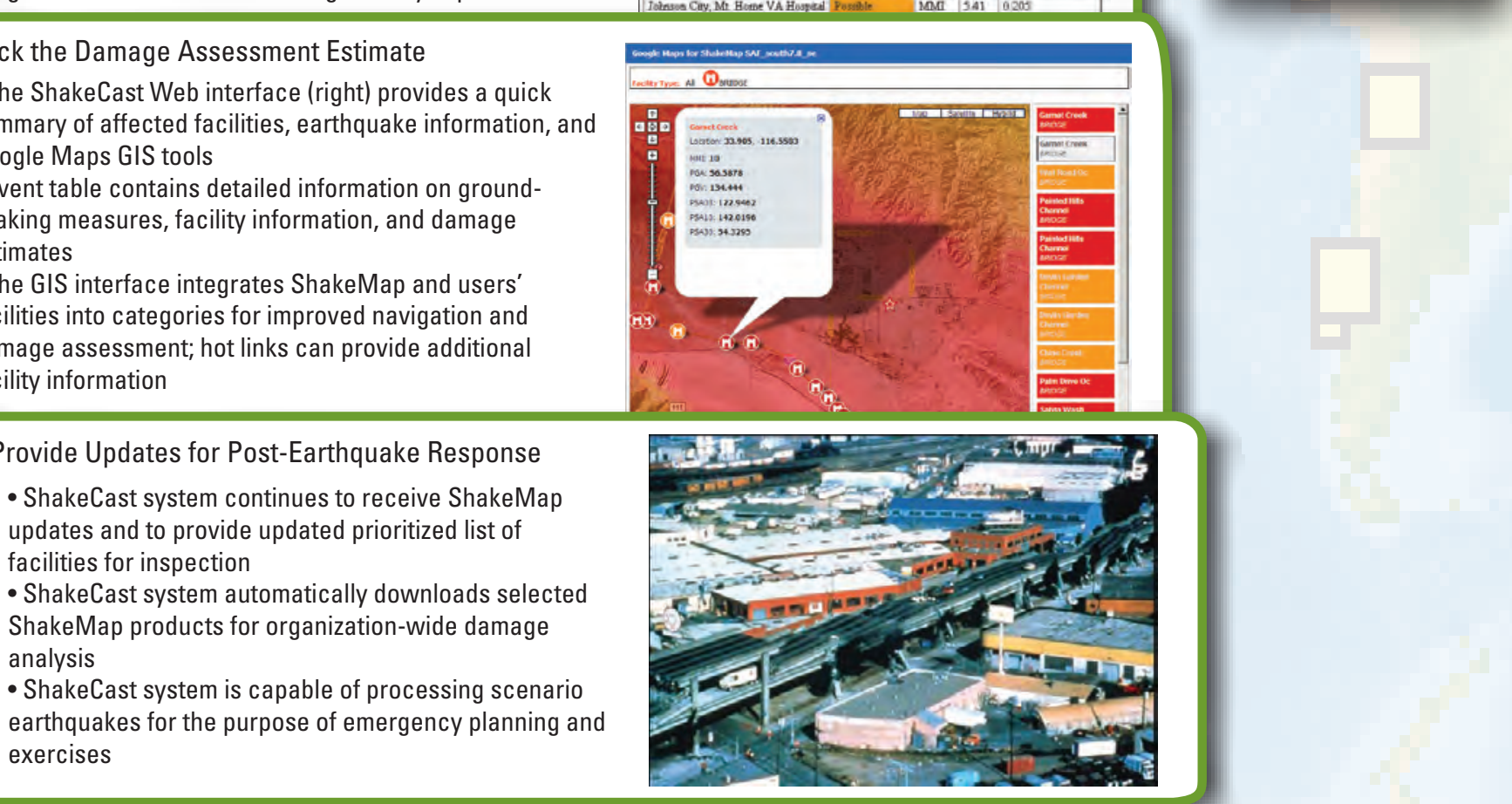

Figure 3. ShakeCast overview from the users' perspective. 


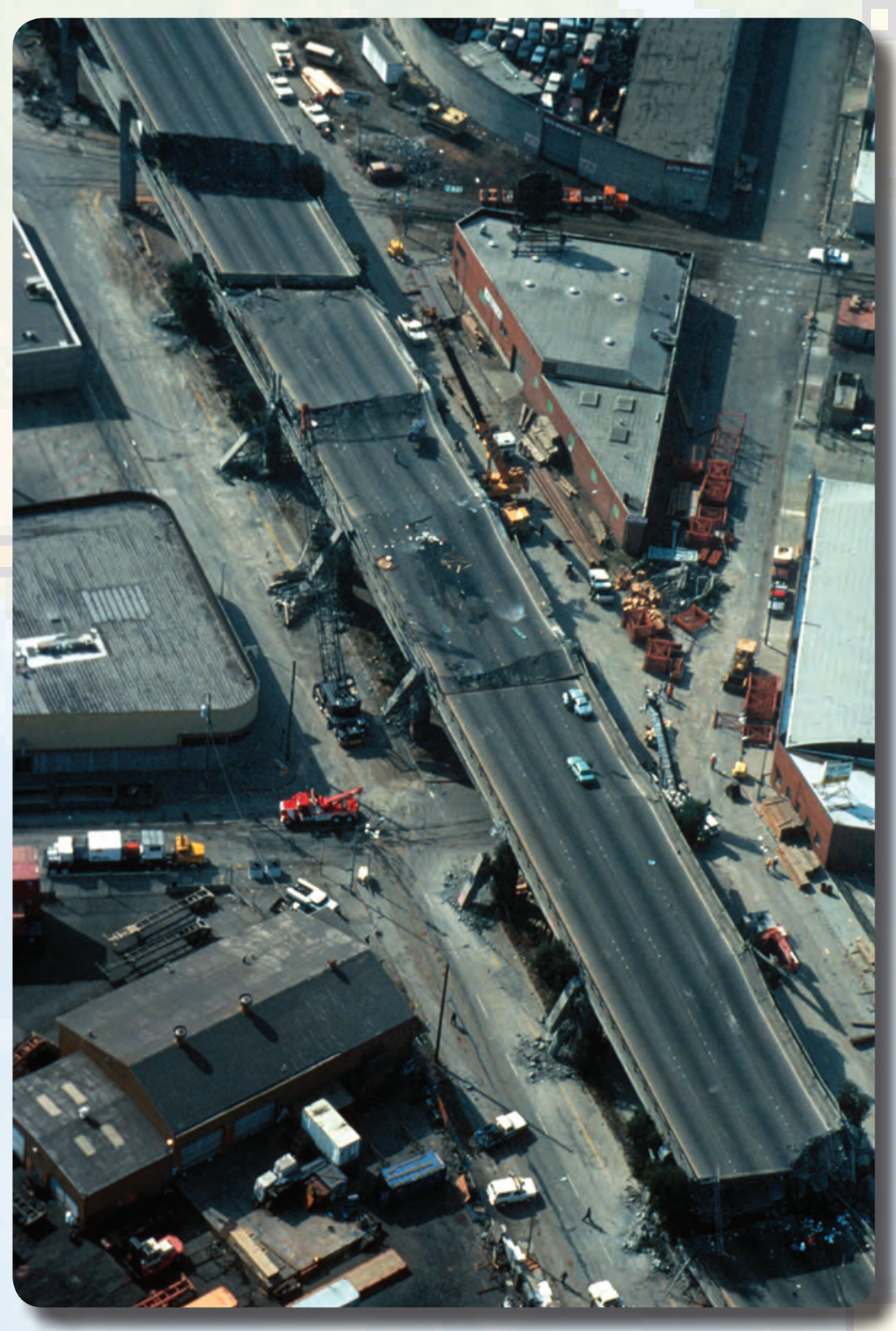

Collapsed section of the Cypress viaduct of Interstate 880 in Oakland following the magnitude 6.9 earthquake in 1989 in Loma Prieta, California.
- Expanded GIS support for common data exchange formats and application programming interfaces (APIs) (currently supports data export via user-defined templates).

- Support for UNIX, Mac, and LINUX operating systems (currently runs on Microsoft Windows).

- Compute and visualize uncertainties in ground shaking and damage likelihood.

- Improved re-notification logic, allowing flexibility in conditions for re-alerting (for example, if damage state changes for one or more facilities).

Optional, automatic updates of the software will be provided by the RSS feed from USGS Web servers.

\section{Sources of Additional Information}

\section{On the Web:}

ShakeMap: http://earthquake.usgs.gov/shakemap/ ShakeCast Software: http://earthquake.usgs.gov/shakecast/

\section{Contacts:}

Kuo-Wan Lin, USGS, Golden, CO, klin@usgs.gov, (303) 273-8563 David Wald, USGS, Golden, CO, wald@usgs.gov, (303) 273-8441

\section{Acknowledgments}

USGS contracted with Gatekeeper Systems, Inc., Pasadena, California, to help develop the prototype ShakeCast system (Version 1.0). Earlier funding for ShakeCast was provided by the American Lifelines Alliance (ALA), and it is now supported by the U.S. Geological Survey under the Advanced National Seismic System (ANSS). Motivation and support for the development of Version 2 of ShakeCast was provided by the California Department of Transportation (Caltrans) under the coordination efforts of Loren Turner.

Any use of trade, product, or firm names is for descriptive purposes only and does not imply endorsement by the U.S. Government. 